\title{
ON GENERICITY AND COMPLEMENTS OF MEASURE ZERO SETS IN FUNCTION SPACES
}

\author{
D. REBHUHN ${ }^{1}$
}

\begin{abstract}
Generic properties of function spaces have been of particular interest in dynamical systems and singularity theory. The underlying assumption has been that the complement of a dense $G_{8}$ set is sparse enough to be considered unlikely. Nevertheless, in infinite dimensional spaces, even dense $G_{\delta}$ 's may have measure zero. Since there is no one canonical measure on an infinite dimensional Fréchet space, notions of measure zero have not often been considered. Here we use a notion of Haar measure zero on abelian Polish groups due to Christensen [1]. We show that those sections of a finite dimensional vector bundle over a compact manifold whose jets are transverse to a submanifold of the jet bundle are complements of sets of Haar measure zero.
\end{abstract}

An abelian Polish group $G$ is an abelian topological group with group operation + such that $G$ has a separable Hausdorff topology and such that there exists at least one complete metric on $d$ inducing the given topology.

A universally measurable subset of $G$ is a subset of $G$ that is measurable for every probability measure defined on the Borel sets of $G$.

We will say that a universally measurable subset $A$ of $G$ has Haar measure zero if there is a (nonunique) probability measure $d u$ on $G$, called a testing measure, such that for any $g \in G, \int_{G} \chi_{A+g} d u=0$. Here $A+g$ is the $g$-translate of $A$, and for any $S \subset G, \chi_{s}$ is the characteristic function of $S$. That is, the set $A$ has Haar measure zero if $A$ and all its translates have measure zero with respect to the testing measure.

We observe without proof that if $G$ were a locally compact group with Haar measure $d h$, then the notion we have defined is equivalent to saying that $\int_{G} \chi_{A} d h=0$. We also observe that the countable union of Haar measure zero sets is a set of Haar measure zero. The justification for interest in this notion of measure zero is that it seems to be a suitable one for doing calculus on abelian Polish groups. In particular, there is a generalization of a theorem of Rademacher saying that Lipschitz mappings between certain abelian Polish groups have directional derivatives linear in the direction a.e. in this sense.

Received by the editors May 23, 1977.

AMS (MOS) subject classifications (1970). Primary 58D99; Secondary 28A70.

Key words and phrases. Space of sections of a bundle, transversality, genericity, Polish group, Haar measure zero.

${ }^{1}$ Research supported by a grant from the Radcliffe Institute. 
See [1] for details and further references.

Let $M$ be a compact, connected $C^{\infty}$ manifold. Let $B$ be a vector bundle over $M$ with finite dimensional fiber. We will use the symbol $C^{\infty}(B)$ to denote the set of $C^{\infty}$ sections of $B$ with addition of sections as the group operation. For any nonnegative integer $k$, we will let $J^{k}(B)$ denote the $k$-jet bundle of sections from $M$ to $B$. There is a well-known transversality theorem that says that if $W$ is a submanifold of $J^{k}(B)$, then there is a residual subset $R$ of $G$ such that if $g \in R$ then $j^{k} g: M \rightarrow J^{k}(B)$ is transversal to $W$ (see [2, II. 4.9]). Here $j^{k} g$ is the $k$-jet mapping of $g$. Our main result will be

I. THEOREM. Let $G=C^{\infty}(B)$. Let $W$ be a submanifold of $J^{k}(B)$ and let $R$ be the subset of those sections $g \in G$ such that $j^{k}(g): M \rightarrow J^{k}(B)$ is transverse to $W$. Then $G$ is an abelian Polish group and $R$ is the complement of a set of Haar measure zero in $G$.

To prove the theorem, we will need some lemmas.

II. LEMMA. $G$ is separable.

Proof of Lemma II. Since $M$ is compact, we can find a positive integer $p$ and for each $i=1,2, \ldots, p$ we can find a $C^{\infty}$ function $\phi_{i}: M \rightarrow[0,1]$ such that

1. (i) $\phi_{i}$ has compact support $C_{i}$,

(ii) $C_{i} \subset U_{i} \subset M$, where $U_{i}$ is open and diffeomorphic to an open subset of $\mathbf{R}^{m}$, and

(iii) $\sum_{i=1}^{p} \phi_{i}(x)=1$ for any $x \in M$.

As a result, any section $g \in G$ can be written as a finite sum $g=\Sigma \phi_{i} g$. Here $\phi_{i} g$ is a $C^{\infty}$ section of $B$ with compact support in $C_{i}$.

We will show the existence of a countable set of sections that are dense in the set $G_{i}=\left\{\phi_{i} g: g \in G\right\}$. This will complete the proof of Lemma II.

We may, without loss of generality, assume that the restriction of $B$ to $U_{i}$ is trivial. That is, $\left.B\right|_{U_{i}} \approx U_{i} \times \mathbf{R}^{n}$ for some integer $n$. In particular, we may identify $U_{i}$ with an open subset of $\mathbf{R}^{m}$ and identify sections of $\left.B\right|_{U_{i}}$ with $C^{\infty}$ maps from $U_{i}$ to $\mathbf{R}^{n}$. By the Weierstrass approximation theorem, see [3], those maps whose component functions are polynomials with rational coefficients can be used to uniformly approximate any other map up to order $k$ in the $C^{k}$ sup norm on $C_{i}$. In particular, if we denote those countably many polynomial maps by $\left\{p_{j}: j \in N\right\}$, then $\left\{\phi_{i} p_{j}: j \in N\right\}$ is dense in $G_{i}$. This proves the lemma.

II. Lemma. There is a complete metric $d$ on $G$ that induces the Whitney $C^{\infty}$ topology.

Proof. The Whitney $C^{\infty}$ topology is the coarsest topology which is finer than the Whitney $C^{k}$ topology for any finite $k$. If $G^{k}=C^{k}(B)$ is the set of $C^{k}$ sections of $B$ in the Whitney $C^{k}$ topology, then there is a complete metric $d^{k}$ on $G^{k}$ (see [2, p. 43]). Since $G \subset G^{k}$, there is an induced metric on $G$ which we will also call $d^{k}$. If $g, g^{\prime} \in G$, we will define a metric on $G$ by 


$$
d\left(g, g^{\prime}\right)=\sum_{k=0}^{\infty} \frac{1}{2^{k}} \frac{d^{k}\left(g, g^{\prime}\right)}{1+d^{k}\left(g, g^{\prime}\right)}
$$

It is not hard to see that $d$ is complete and induces the proper topology on $G$.

Now let us prove Theorem I. As in [2, II. 4.9], we choose a countable open cover of $W$ by open sets $W_{1}, W_{2}, \ldots$ such that:

2. (i) the closure of $W_{i}$ in $J^{k}(B)$ is contained in $W$;

(ii) $\bar{W}_{i}$ is compact;

(iii) there is a chart $U_{i}$ on $M$ such that $\left.B\right|_{U_{i}} \approx U_{i} \times \mathbf{R}^{n}$ for some integer $n$ and such that $\pi\left(\bar{W}_{i}\right) \subset U_{i} \times \mathbf{R}^{n}$, where $\pi: J^{k}(B) \rightarrow B$ is the natural projection mapping; and

(iv) $\bar{U}_{i}$ is compact.

If we let $T_{i}=\left\{g \in G: j^{k} g \uparrow W\right.$ on $\left.\pi \bar{W}_{i}\right\}$, then just as in [2], each $T_{i}$ is open and dense and $R=\cap T_{i}$. Let us show that for each $i$, the set $C T_{i}$, the complement of $T_{i}$, is a set of Haar measure zero.

Let $\pi_{1}$ be the projection of $J^{k}(B)$ onto $M$. Note that $\bar{W}_{i} \subset \pi_{1}^{-1}\left(U_{i}\right)$. We can always assume that we have chosen the $W_{i}$ and $U_{i}$ small enough so that there exist a finite integer $q$ and $q$ sections $g_{1}, g_{2}, \ldots, g_{q} \in G$ such that:

3. (i) for each $g \in G$ we can define a map $\bar{g}: \mathbf{R}^{q} \rightarrow G$ by $\bar{g}\left(s_{1}, \ldots, s_{q}\right)(x)=$ $\left(s_{1} g_{1}+\cdots+s_{q} g_{q}+g\right)(x)$;

(ii) the map $\underline{g}$ from $\mathbf{R}^{q} \times M \rightarrow J^{k}(B)$, defined by $\underline{g}\left(\left(s_{1}, \ldots, s_{q}\right), x\right)=$ $j^{k}\left(\bar{g}\left(s_{1}, \ldots, s_{q}\right)\right)(\bar{x})$, is onto $\pi_{1}^{-1}\left(U_{i}\right)$;

(iii) for each fixed $x \in U_{i}$, the map $\underline{g}((\cdots), x)$ from $\mathbf{R}^{q}$ to $J^{k}(B)$ is surjective at each point in its domain.

To see that such $g_{1}, \ldots, g_{q}$ can exist, we identify $U_{i}$ with a neighborhood of zero in $\mathbf{R}^{m}$ and let $g_{1}, \ldots, g_{q}$ be the set of all distinct maps to $\mathbf{R}^{n}$ with only one nonzero entry that is a monomial of order $\leqslant k$ in $m$ variables.

Let $S=\overline{0}\left(\mathbf{R}^{q}\right)$ be the image of $\mathbf{R}^{q}$ in $G$. Here 0 is the zero section of $B$. We will induce a probability measure on $S$ by using a probability measure on $\mathbf{R}^{q}$ which assigns probability zero to sets of Lebesgue measure zero. If $R \subset S$, we define the measure of $R$ to be the measure of $(\overline{0})^{-1}(R)$. If $R \subset G$, but $R$ is not a subset of $S$, we define the measure of $R$ to be the measure of $R \cap S$.

We wish to show that $C T_{i}$ has measure zero with respect to the testing measure we have just defined.

By hypotheses 3(i)-(iii), for any $g \in G$, the map $\underline{g}: \mathbf{R}^{q} \times M \rightarrow J^{k}(B)$ is transversal to $W$ on $U_{i}$. By Lemma II.4.6 of [2], the set $A_{g}$ of points $\left(s_{1}, \ldots, s_{q}\right) \in \mathbf{R}^{q}$ such that $\underline{g}\left(\left(s_{1}, \ldots, s_{q}\right), \cdot\right)$ is transversal to $W$ on $U_{i}$ is open and dense in $\mathbf{R}^{q}$. Careful reading of the proof of the lemma shows that $\mathbf{R}^{q}-A_{g}$ is a set of measure zero.

In particular, $\mathbf{R}^{q}-A_{0}=(\overline{0})^{-1}\left(C T_{i} \cap S\right)$ and $\mathbf{R}^{q}-A_{-g}=\overline{0}^{-1}\left[\left(C T_{i}+g\right)\right.$ $\cap S]=(-\bar{g})^{-1}\left(C T_{i} \cap S\right)$. That is $C T_{i}$ and all its translates have measure zero. Thus $C T_{i}$ must be a set of Haar measure zero.

REMARKS. 1. The same proof shows that if $W$ is a submanifold of $J^{k}(B)$, then the elements of $G^{k+1}$ that are transversal to $W$ are the complement of a 
set of Haar measure zero. Recall that $G^{k+1}$ is the set of $C^{k+1}$ sections of $B$ in the Whitney $C^{k+1}$ topology.

2. If we are considering the set of $C^{\infty}$ maps from compact connected $M$ to $N$ in the $C^{\infty}$ topology, these form a Fréchet manifold (see [2, III.1.11]). Given $f \in C^{\infty}(M, N)$, we can find a neighborhood $U$ of $f$ in $C^{\infty}(M, N)$ that can be identified with an open subset of a Fréchet space and show that those elements of $U$ transversal to $W$ on $M$ are the complement of a set of Haar measure zero.

3. Even if $M$ is noncompact, at first it seems that the definition of Haar measure zero still applies to sections of $C^{\infty}(B)$ where $B$ is a vector bundle over $M$. In this case, Christensen's proof that countable union of measure zero sets has measure zero does not hold. Also, if $M$ is noncompact, $C^{\infty}(B)$ is not separable and the generalization of Rademacher's theorem may not hold.

ACKNOWLEDGEMENTS. I would like to thank R. Dudley for useful conversations, the Radcliffe Institute for moral and financial support, and M.I.T. for hospitality extended while I completed this work.

\section{REFERENCES}

1. J. P. R. Christensen, Topology and Borel structure, American Elsevier, New York, 1974.

2. M. Golubitsky and V. Guillemin, Stable mappings and their singularities, Springer-Verlag, New York, 1973.

3. R. Narasimhan, Analysis on real and complex manifolds, North-Holland, Amsterdam, 1968.

Radcliffe Institute, 3 James St., Cambridge, Massachusetts 02138

Current address: Department of Mathematics, Vassar College, Poughkeepsie, New York 12601 